\title{
Multi Agent Based Congestion Control in VANETs
}

\author{
Ramesh B. Koti and Mahabaleshwar S. K.
}

\begin{abstract}
Vehicular Ad hoc Networks (VANET) play an important role in future on road Vehicle-to-Vehicle communication systems and related applications like self-organizing, acquiring traffic information using sensors and disseminating it to the nearby vehicles, which are based on broadcast/geocast transmission schemes. It is a challenging task to manage networks in a dynamic environment due to the characteristic of high mobility rate, high relative speed, different driver behaviors and unpredictable link failure occurrences. One of the main criteria in VANETs that has attracted the researchers' consideration is congestion control. Congestion is a major problem all over world, resulting in wastage of valuable time and money. In this paper, we propose multi agent based congestion control scheme, which intelligently acquires the status of the neighboring node information and decides the congestion free path for the safe delivery of packets. Multi agent scheme consists of set of static and mobile agents. Proposed approach guarantees the reliability and timely delivery of messages. Multi agent based scheme exhibits better bandwidth usage and packet delivery ratio and minimizes the latency
\end{abstract}

Index Terms-VANETs, multi agent, latency, bandwidth, congestion control.

\section{INTRODUCTION}

Vehicular ad hoc network (VANET) is a promising form of mobile ad hoc network, in which vehicles are equipped with radios for inter-vehicle communication [1]. As an important part of intelligent transportation system, the primary task of the VANET is to aggregate and facilitate information to and from all the neighboring vehicles for situation or threat assessment. Congestion is one of the key issues, which can restrict the network performance, and it is no exception for VANETs. Congestion control mechanisms include three phases: congestion detection, congestion notification and rate adjustment. Congestion detection is the first stage of congestion control mechanisms because the available resources are limited, and the network topology and

node density change over time, applying conventional congestion detection protocols to VANETs can be problematic.VANET raises several interesting issues in regard to media access control (MAC), data aggregation, data validation, data dissemination, routing,network congestion, performance analysis, privacy and security [2]-[5].

\section{LITERATURE SURVEY}

The work given in [6] scans the messages queues and

Manuscript received July 10, 2013; revised October 28, 2013.

The authors are with the Electronics and Communication Department, Basaveshwar Engineering College, Bagalkot-587102, Karnataka, India (e-mail: ramesh_koti@sify.com, mahabalesh_sk@yahoo.co.in). monitor channel communications based on defined threshold. Forward phase is responsible to deliver emergency messages to the adjacent node and also the farthest node for fast propagation. A scheme for congestion control by sending Beacon Adaptive methods of setting the rate or range of transmission, instead of using the fixed values of $10 \mathrm{~Hz}$ and $250 \mathrm{~m}$ is discussed in [7]. The beacon messages are used by the neighboring vehicles (nodes) to be aware of their environment as well as preventing potential dangers. The event-driven safety messages are generated when an abnormal condition or an imminent danger is detected and are disseminated within a certain range with higher priority.

The control algorithm uses an application specific utility unction and encodes the quantitative utility information in each transmitted data packet in a transparent way for all users within a local environment [8]. A decentralized algorithm then calculates the "average utility value" of each individual node based on the utility of its data packets and assigns a share of the available data rate proportional to the relative priority.

In the research work [9], vehicles listen for the neighbors beacons and decide that it is in congestion. All the vehicles are equipped with short range wireless devices and each node has a unique ID which is an Integer. Each device does the beaconing in random intervals. Here each vehicle builds a speed map based on the speed of other vehicles in its vicinity and transmits it to neighboring vehicle. A traffic information sharing and route selection procedure to address the problem of vehicle traffic congestion is presented in [10].

\section{Multi Agent Based Congestion Control}

Multi agent based approach controls the congestion that is likely to occur because of the heavy usage of bandwidth, Buffer \& cache memory in VANET.Agents are used to collect and control intelligently of these information parameters. In this section, we discuss network environment and proposed scheme.

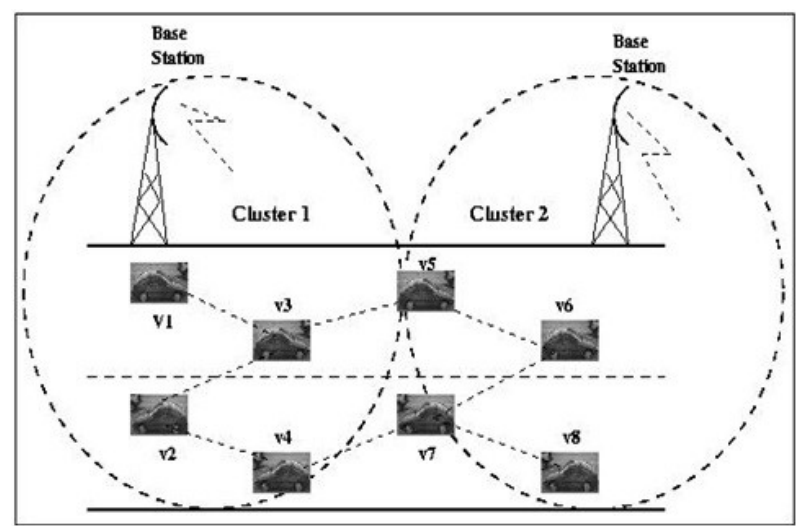

Fig. 1. Network environment. 


\section{A. Network Environment}

Proposed congestion control scheme considers a VANET scenario as shown in Fig. 1. We assume some communicating devices called vehicles, spread throughout the network (Ad hoc) randomly. Here vehicles are termed as nodes. Each node is limited by its transmission range, mobility, buffer memory, cache memory \& bandwidth utilized. And also each node incorporates an agent code which is mobile agent. Source nodes always use one or more Intermediate nodes to communicate with the intended node. The nodes, which lie within the transmission range of a node, are said to be neighboring nodes for that particular node.

The node, which is in need to communicate with some other node, is termed as source node, with which the source node wants to communicate is termed as destination node. Each cluster is associated with the nodes which can form the direct connection among themselves. There will be a link between clusters by the neighboring nodes. For any source to destination there may be many paths to reach. Any node in any cluster can reach any node in the other cluster through the intermediate nodes.

\section{B. Proposed Scheme}

Our scheme has two aspects, identifying congestion and controlling the congestion. We assume all the vehicles are equipped with short-range wireless devices and each node has a unique ID, which is an integer. Static agent has information about their status. Agents will get this information to parent node. Nodes listen for the neighbor's beacons and decide that it is in congestion. Likewise all the nodes decide their congestion state. Once the node has decided it is in congestion it finds an alternative path, which has efficient performance in its parameters.

Proposed algorithm will select the node having the largest space for the buffer, cache memory, smallest bandwidth used Mobility has an impact on the performance of the system which also be considered at different factors. Each node thus finds an efficient neighboring node to reach to the destination. We deployed the nodes randomly with code each node will have the total of its sub tree. The model gives the table of values, which contain the information about nodes, later the destination is to find with help of this table. Each node will have its current neighbors list, previous neighbors list and the last set of beacons received along with the mobility, bandwidth, buffer \& cache memory used.

Mobile agent brings the information from the node and dissemination will be based on the available efficient route in terms of the parameters like bandwidth, cache \& buffer memory. A node can be in one of several states. Initially it is in an unknown state and then it transits to congestion state where it gets added to the tree and becomes a Child or a Parent and finally a Root.

Each node will be assigned with some random parameter values using random function. Connection will be made between the clusters, defining the source node and destination node, and finding the all available path from source to destination. Among available routes efficient path is chosen based on the values of the parameters. The congestion factor ( $i)$ is expressed as by the equation (1). Setting the threshold value for congestion free path, we can determine the two ways of discovering the congestion.

$$
i=\mathrm{M} / \mathrm{BCBw}
$$

where $M=$ mobility of the node

$B w=$ bandwidth utilized

$B=$ buffer memory

$C=$ cache memory

\section{Simulation}

The proposed scheme has been simulated in various network scenarios using $\mathrm{C}++$ language. We consider $n$ number of nodes moving in a fixed region of length $A \mathrm{Km}$. and breadth $B \mathrm{Km}$.Communication coverage area for each sensor node is considered as a Vcom meters. Coverage area around each node has a bandwidth $B W$ shared among neighbors. Mobility factor for each sensor node is in between the range of $I$ to $J$ meters per second.

\section{A. Simulation Inputs}

The simulation input parameters considered are as follows: $\mathrm{n}=10$ to $30, \mathrm{~A}=1000 \mathrm{mts}$., $\mathrm{B}=1000 \mathrm{mts}$., Vcom $=150 \mathrm{mts}$, $\mathrm{BW}=1000 \mathrm{Kbps}, \mathrm{I}=10, \mathrm{~J}=30$ meters per second.

\section{B. Performance Metrics}

Some of performance metrics evaluated are packet delivery ratio, bandwidth utilized, latency and control overhead.

Packet Delivery Ratio: Packet delivery ratio is the ratio of the packets received to the packets generated. It is expressed in terms of percentage.

Bandwidth utilized: It is the ratio of total amount of band width utilized out of the total amount of bandwidth available by the vehicles participating in the network activities. It is expressed in terms of Kbps.

Latency: It is the time taken for the destination vehicle to receive the packets sent by the source vehicle. It is expressed in terms of milli seconds.

\section{Result Analysis}

The relation between packet delivery ratio (PDR) and number of vehicles is presented in Fig. 2. PDR decreases gradually as the number of nodes increases. It is represented for ifferent mobility.

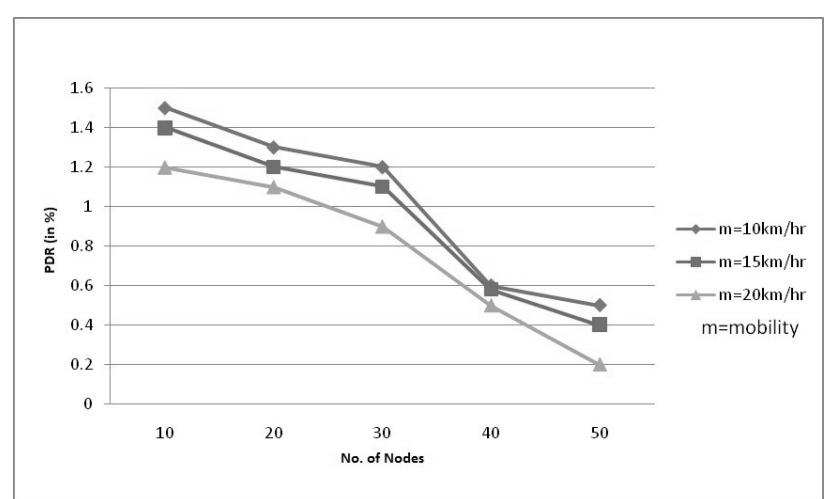

Fig. 2. Packet delivery ratio vs. no. of nodes.

Fig. 3 gives the relation between bandwidth utilized by varying the number of nodes. We observe that the bandwidth 
usage increases with increase in the number of nodes.

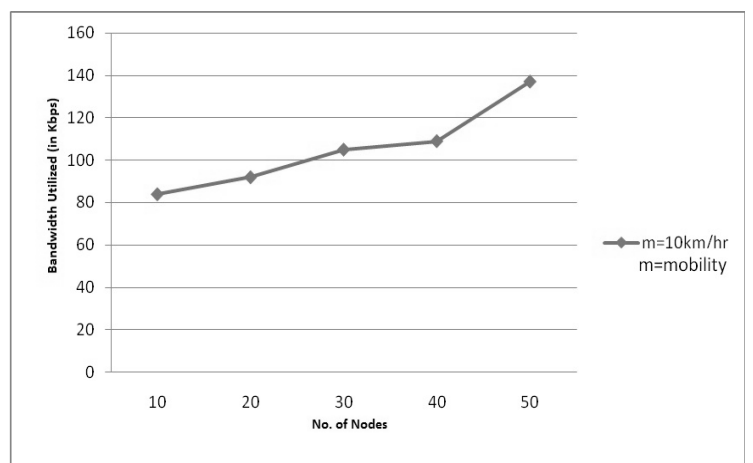

Fig. 3. Bandwidth utilized vs. no. of nodes

Fig. 4 gives the relation between Latency with respect to the variation in the number of nodes. To take the source packet to the destination amount of time it takes increases almost linearly with the number of nodes. As node density increases the latency increases.

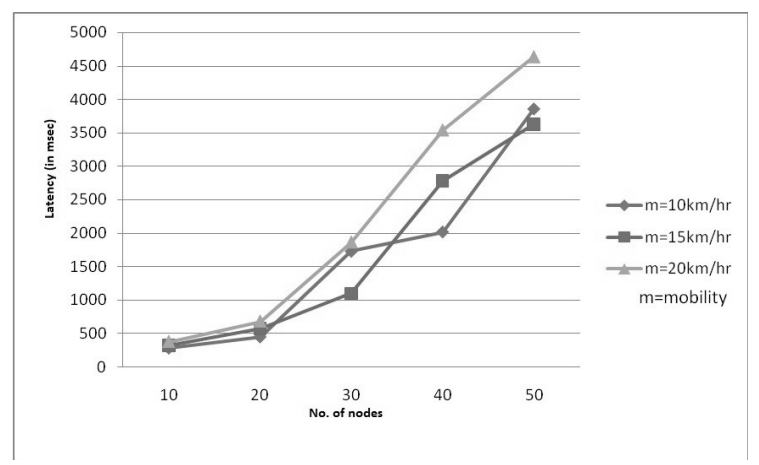

Fig. 4. Latency vs. no. of nodes.

\section{CONCLUSION}

Congestion control is a challenging issue for VANETs. Due to the mainly broadcast/geocast oriented communication and the highly dynamic network topology, conventional mechanisms such as per-flow fair queuing are difficult to apply. In this paper, we propose multi agent based congestion control in VANETs based on parameters band width utilized, buffer used and cache memory used. The proposed scheme has been simulated in various network scenarios for testing the performance and operation effectiveness. Some of the performance parameters analyzed are packet delivery ratio, latency and bandwidth usage.

The proposed work can be compared and simulated with many other existing congestion control mechanisms.

\section{ACKNOWLEDGMENT}

We are very much thankful to TEQIP phase II of World Bank of Basaveshwar Engineering College (Autonomous), Bagalkot, Karnataka, India for sponsoring the Registartion, TA and DA expenses for attending 5th International
Conference on Information and Multimedia Technology (ICIMT 2013), 6-7 December 2013, Sydney, Australia.

\section{REFERENCES}

[1] M. S. Kakkasageri and S. S. Manvi, "Safety information management in vehicular ad-hoc networks: A review," Journal of Network and Computer Applications, Elsevier, pp. 25-30, 2003.

[2] C. P. Hridya, N. S. Vibha, M. S. Kakkasageri, S. S. Manvi, and P. I. Basarkod, "A tutorial on cognitive agent architectures," IETE Journal of Education, vol. 53, no. 1, pp. 21-37, Jan.-Jun. 2012.

[3] S. S. Manvi and M. S. Kakkasageri, "Issues in mobile ad hoc networks for vehicular communication," IETE Technical Review, vol. 25, no. 2, pp. 59-72, 2008.

[4] M. S. Kakkasageri and S. S. Manvi, "Safety information aggregation in VANETs using vehicle beliefs," in Proc. IEEE $5^{\text {th }}$ International Symposium on Advanced Networks and Telecommunication Systems (ANTS 2011), Bangalore, India, December 19-21, 2011.

[5] S. S. Manvi, M. S. Kakkasageri, and J. Pitt, " Information search and access in vehicular Ad-hoc networks (VANETs): An agent based approach," in Proc. International Conference on Communication in Computing (CIC-2007), The 2007 World Congress in Computer Science, Computer Engineering, and Applied Computing, Las Vegas, Nevada, USA, pp. 23-29, June 25-28, 2007.

[6] M. Y. Darus and K. A. Bakar, "Congestion control framework for emergency messages in VANETs," International Journal of Research and Reviews in Computer Science (IJRRCS), pp.199-203, 2008.

[7] M. R. J. Sattari, R. M. Noor, and H. Keshavarz. A Taxonomy for congestion control algorithms in vehicular ad hoc networks. [Online]. Available: http://www. arxiv.org/ftp/arxiv/papers/1207/1207.3943.pdf

[8] L. Wischhof and H. Rohling "Congestion control in vehicular ad hoc networks," in Proc. IEEE International Conference on Vehicular Electronics and Safety, pp. 58-63, 2005.

[9] M. F. Fahmy and D. N. Ranasinghe, "Discovering automobile congestion and volume using VANET's," in Proc. 8th International Conference on ITS Telecommunications, pp. 367-372, 2008.

[10] B. K. Mohandas, R. Liscano, and O. W. W. Yang, "Vehicle traffic congestion management in vehicular ad-hoc networks," in Proc. $L C N$ 2009 , pp. $655-660$

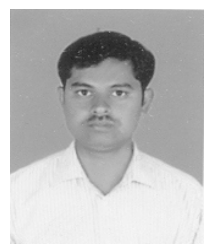

Ramesh B. Koti completed his B. E in electronics and communication engineering from Visvesvaraya Technological University Belgaum, India and pursuing his M.Tech in Digital Electronics and Communication from Visvesvaraya Technological University Belgaum, India. Presently, he is working as faculty in Department of Electronics and Communication Engineering, Basaveshwar Engineering College, Bagalkot, Karnataka, India. He has published 1 international conference papers. His areas of interest are wireless networks, especially vehicular ad hoc networks, and sensor networks. He is a member of IETE India.

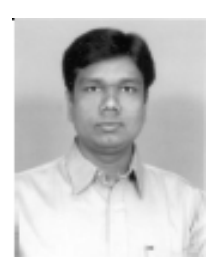

Mahabaleshwar S. K. completed his B. E in electronics and communication engineering from Karnataka University Dharwad, India, M.Tech, and Ph. D. from Visvesvaraya Technological University Belgaum, India. Presently, he is working as Associate Professor in Department of Electronics and Communication Engineering, Basaveshwar Engineering College, Bagalkot, Karnataka, India. He has published 20 national and international conference papers and 8 national and international journals. Recently, he has coauthored a book on Wireless and Mobile Networks: Concepts and Protocols, published by Wiley-India. His areas of interest are wireless networks, especially vehicular ad hoc networks, and sensor networks. He is a member of IEEE USA, IETE India. 